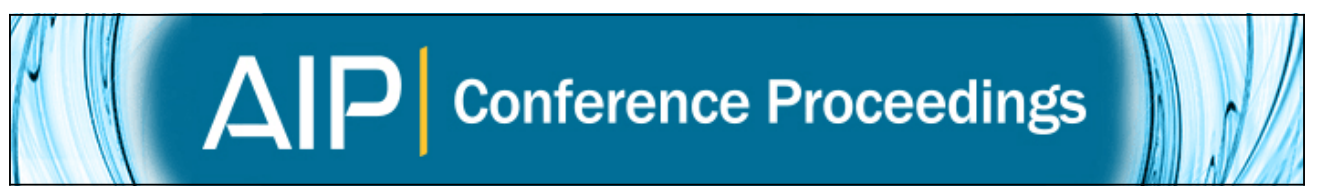

\title{
Probing dark gamma-ray bursts with neutrinos
}

Shunsaku Horiuchi and Shin'ichiro Ando

Citation: AIP Conference Proceedings 1178, 97 (2009); doi: 10.1063/1.3264563

View online: http://dx.doi.org/10.1063/1.3264563

View Table of Contents:

http://scitation.aip.org/content/aip/proceeding/aipcp/1178?ver=pdfcov

Published by the AIP Publishing

Articles you may be interested in

Multi-messenger observations of gamma-ray bursts

AIP Conf. Proc. 1358, 365 (2011); 10.1063/1.3621806

The Collapsar Model for Gamma-Ray Bursts

AIP Conf. Proc. 727, 343 (2004); 10.1063/1.1810862

Possible Class of Nearby Gamma-Ray Burst / Gravitational Wave Sources

AIP Conf. Proc. 686, 74 (2003); 10.1063/1.1629417

Gravitational Waves from Gamma-Ray Bursts

AIP Conf. Proc. 686, 67 (2003); 10.1063/1.1629416

Gamma-ray bursts and gravitational waves

AIP Conf. Proc. 575, 143 (2001); 10.1063/1.1387307 


\title{
Probing dark gamma-ray bursts with neutrinos
}

\author{
Shunsaku Horiuchi* and Shin'ichiro Ando ${ }^{\dagger}$ \\ *Institute for the Physics and Mathematics of the Universe, the University of Tokyo, 5-1-5, \\ Kashiwanoha, Kashiwa, Chiba 277-8582, Japan \\ ${ }^{\dagger}$ California Institute of Technology, Mail Code 130-33, Pasadena, CA 91125
}

\begin{abstract}
Highly relativistic jets are a key element of current gamma-ray burst (GRB) models, where the jet kinetic energy is converted to radiation energy at optically thin shocks. Mildly relativistic jets with smaller Lorentz factors are typically optically thick to gamma rays, and do not produce the spectacular GRB phenomenon. Jets which stall inside the progenitor similarly do not produce a GRB. However, various studies suggest that these jets are more common than GRBproducing jets. Here we report on our study of high-energy neutrino emission from these hidden jets. We describe the detection prospects with near-future neutrino detectors, and discuss how the presence of jets can be studied with neutrinos. The neutrino horizon for hidden jets is of order 10 $\mathrm{Mpc}$, a volume which contains at least a few supernova per year.
\end{abstract}

Keywords: Supernovae, Gamma-ray bursts, Neutrinos

PACS: $97.60 . \mathrm{Bw} ; 98.70 . \mathrm{Rz} ; 95.85 . \mathrm{Ry}$

\section{INTRODUCTION}

The leading model of GRBs involves a relativistic $\left(\Gamma_{j} \sim 100\right.$, where $\Gamma_{j}$ is the jet Lorentz factor) fireball jet, where the observed gamma rays are produced by radiation from Fermi-accelerated electrons in optically thin shocks (for reviews see e.g. [1,2]). In the most popular model for the more common long-duration GRBs, the so-called collapsar model, the core of a massive star collapses to a black hole or neutron star, driving a highly relativistic jet which breaks out of the star [3,4]. The GRB phenomena occurs when shells within the jet, travelling at different velocities, collide with each other, thereby producing shocks and subsequent pulses of radiation. With $\Gamma_{j}>100$, the jet is optically thin to gamma ray radiation at the internal shock radius; a GRB is possible.

Inferring from the observed rate of GRBs, highly relativistic $\left(\Gamma_{j} \sim 100\right)$ jets are rare events and occur in perhaps $10^{-4}-10^{-3}$ of core-collapse supernovae, taking into account jet beaming effects [5]. The rarity should reflect the various requirements for a GRB to occur; for example, that the baryon content of the jet must be very small, $\sim 10^{-6} \mathrm{M}_{\odot}$. An intriguing question to ask is whether jets with more baryons exist in nature. However, a baryon rich jet cannot be studied from its prompt gamma-ray signature, because the optical depth of the source is expected to be much larger than 1 . Neutrinos would be the only prompt signature of these hidden structures.

While not direct, some progress have been made in searching for mildly relativistic baryon rich jets from their late-time manifestations. For example, recent observations of late-time radio afterglows in otherwise normal core-collapse supernovae have suggested that the fraction of core-collapse supernovae endowed with jets could be as high as a few percent $[6,5,7,8]$. Also, recent detection of low-luminosity GRB 060218 suggests

\footnotetext{
CP1178, The Dark Side of the Universe edited by $\mathrm{C}$. Balázs and $\mathrm{F}$. Wang

(C) 2009 American Institute of Physics 978-0-7354-0719-0/09/\$25.00
} 
TABLE 1. Jet definitions and their observables. Successful highly relativistic jets are the leading model for GRBs. The successful but mildly relativistic jet and the choked jets are all typically dark in gamma rays. Entry "this work" represents our specific focus.

\begin{tabular}{ccccc}
\hline \multicolumn{4}{c}{ Choked } & Successful \\
& $\Gamma_{j}=10$ & $\Gamma_{j}=100$ & $\Gamma_{j}=10$ & $\Gamma_{j}=100$ \\
\hline$\gamma$ rays & dark & dark & typically dark & bright \\
$v$ & this work & this work & this work; see also $[14,15]$ & e.e., [16] \\
\hline
\end{tabular}

from detection rates that low-luminosity GRBs, with mildly relativistic ejecta, are more common than conventional high-luminosity GRBs by factors as large as $10^{2}[9,10,11$, 12]. These observations support the possibility that a significantly high portion of corecollapse supernovae are endowed with baryon rich, mildly relativistic, jets.

Another type of hidden (in the sense of prompt photon detections) jets are those which fail to break out of the star, and instead stall inside the progenitor photosphere. Socalled "choked" jets, they may be more common than usually appreciated. For example, recent polarimetry observations of supernovae suggest that supernovae are commonly asymmetric, which could be the consequences of hidden jets or ejecta.

Here we report on our investigations of the neutrino signatures of hidden jets. We explicitly note that the definition of hidden jet includes (i) jets that successfully break out of the progenitor but is mildly relativistic, i.e., baryon rich or "dirty" jets, and (ii) jets that stall inside the progenitor star, i.e., "choked" jets. Neutrinos have the important property of opening an unique window on the early epochs of these jets. If confirmed by prompt neutrinos, this would directly show the presence of jets with significant kinetic energy. Data collected over many sources would yield the distribution of jet parameters, and provide important insights on the jet launch mechanism and the supernova-GRB connection. It would also be benefial for timing studies of shock propagation, as well as cross studies with photons and gravitational waves.

We first discuss our treatment of jets, detailing on our calculation of jet propagation and jet classifications. We then discuss the neutrino emission mechanism and neutrino detectability at $1 \mathrm{Km}^{3}$ detectors such as IceCube. We finish with conclusions and propects for the future in the final section.

\section{JET MODELS}

Table 1 summarizes the jets and the scope of this work.

We first discuss the evolution of a relativistic jet as it propagates through and strikes the stellar matter. To do this, we semi-analytically solve the propagation of our parameterized jet through the star and obtain the jet head velocity. Armed with the jet dynamics, we apply our definitions for choked and successful jets and label our fiducial jet models.

Jet dynamics. Generally, two shocks form when a jet strikes external matter: a forward shock that shocks and accelerates the external material to $\Gamma_{h}$, and a reverse shock that shocks and decelerates the head of the jet to a Lorentz factor $\Gamma_{h}$. The 


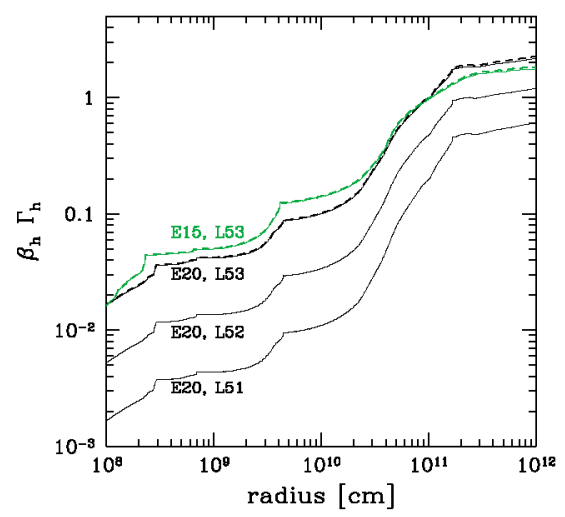

FIGURE 1. Jet head velocity $\beta_{h} \Gamma_{h}$ as a function of radius. E15 and E20 are two progenitor models of initial mass $15 M_{\odot}$ and $20 M_{\odot}$ respectively, as detailed in [13]. L51-L53 denotes the isotropic equivalent luminosity of the jet, $L_{\text {iso }}=10^{51}-10^{53} \mathrm{erg} \mathrm{s}^{-1}$. For the L53 case, two jet Lorentz factors are plotted: mildly relativistic $\left(\Gamma_{j}=10\right.$, solid $)$ and ultrarelativistic $\left(\Gamma_{j}=100\right.$, dashed). For L52 and L51, mildly relativistic jets are shown.

shocked jet and shocked stellar material moving at $\Gamma_{h}<\Gamma_{j}$ are separated by a contact discontinuity, and are in pressure balance. Equations governing the evolution of the shocks are given in various works and are summarized in [13]. The results of solving for the jet propagation are shown in figure 1 , as a function of radius. For all cases, the initial jet head velocity is subrelativistic, then accelerates, reaching mildly relativistic values at $r \sim 10^{11} \mathrm{~cm}$ when the external stellar density drops appreciably near the edge of the He core.

Jet definitions. First we detail on our treatment of choked jets. These jets are progressively shocked and decelerated by the reverse shock, until the entire jet is shocked. Our definition of choked jets is as follows: the duration of the jet is shorter than the jet crossing time to the edge of the He core of the progenitor. From the jet dynamics we can obtain the crossing time, $t_{H e}$, for various jet luminosities and progenitor models, on the assumption that its duration is longer than $t_{\mathrm{He}}$. Then, a jet with duration $T<t_{\mathrm{He}}$ is a choked jet (while $T>t_{H e}$ for a successful jet). This approach is valid since the jet dynamics depend on the luminosity and not on the time-integrated energy.

In principle, the reverse shock exists until it crosses the entire length of the jet. For illustration we calculate the neutrino emission when the reverse shock is at a specific radius: $5 \times 10^{10} \mathrm{~cm}$, which is typically inside the He core. The corresponding time is $\sim 0.7-0.8 t_{\text {He }}$. We note that our choice is partly based on the optical depth of the star to high-energy neutrinos. The neutrino opacity is $\tau_{v}=N_{e x t}(r) \sigma_{v p}\left(\varepsilon_{v}\right)$, where $N_{e x t}$ is the column number density of nuclei in the star and $\sigma_{v p}$ is the sum of neutrino chargedcurrent and neutral-current cross sections, which is approximately $\propto \varepsilon_{V}$ in the energy range of interest. For a $10^{2} \mathrm{GeV}$ neutrino emitted at $r=10^{10} \mathrm{~cm}$, we find that $\tau_{v} \approx 0.68$, and for a $10^{3} \mathrm{GeV}$ neutrino, $\tau_{v} \approx 6$. Thus, only neutrinos with energies $\varepsilon_{v}<10^{2} \mathrm{GeV}$ 


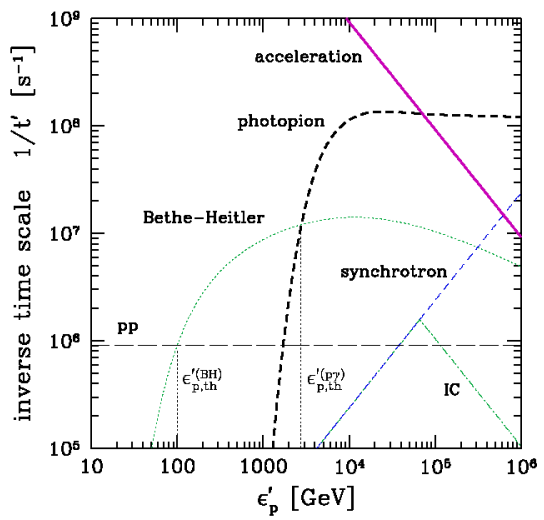

FIGURE 2. Inverse of proton cooling and acceleration time scales, in the shocked jet head frame, as functions of the proton energy. Shown is the instant when the reverse shock is at $r_{\times}=5 \times 10^{10} \mathrm{~cm}$. Cooling mechanisms shown are: synchrotron (blue, thin dashed), inverse-Compton (green, dot-dashed), BetheHeitler (green, dotted), proton-proton (black, long dashed), and photopion (black, thick dashed). Also labeled are threshold energies $\varepsilon_{p, t h}^{\prime(B H)}$ and $\varepsilon_{p, t h}^{\prime(p \gamma)}$ which defines the final spectral shape. Photopion limits the proton energy to $7 \times 10^{4} \mathrm{GeV}$.

can leave the star from $r=10^{10} \mathrm{~cm}$. While this result depends on the stellar density profile assumed, and rotational effects have been neglected in our simple estimate, it sets the general range of stellar radii at which neutrino emission becomes an interesting observable.

Finally, we work with the fiducial choked jet parameterized by $L_{\text {iso }}=10^{52} \mathrm{erg} \mathrm{s}^{-1}$ and $T=34 \mathrm{~s}$, so that the total isotropic energy is $E_{\mathrm{iso}}=3 \times 10^{53} \mathrm{erg}$ (note that $t_{\mathrm{He}}=43 \mathrm{~s}$ ).

\section{NEUTRINO PRODUCTION}

Motivated by GRB studies, we assume that first-order Fermi acceleration is realized, so that a fraction of the jet protons are accelerated to a power-law spectrum with index $s$. In the ultra relativistic limit $s \approx 2.3$, while $s=2$ for a non-relativistic shock [17]. We assume $s=2$ unless otherwise stated.

The maximum proton energy depends on the balance between the acceleration time scale and energy-loss time scales, which in turn depend on various quantities in the vicinity of the shock - for example, the particle density, photon density, and magnetic field strength. We assume that a fraction $\varepsilon_{e}=\varepsilon_{B}=0.1$ of the shocked plasma internal energy (i.e., the kinetic energy that is liberated into internal energy at the shock) is converted to relativistic electrons and magnetic fields, respectively. This yields typical electron densities of $7 \times 10^{25} \mathrm{~cm}^{-3}$ and magnetic fields of $1 \times 10^{10} \mathrm{G}$.

As proton energy-loss mechanims, we consider interactions with photons (pair production, meson production, and inverse-Compton), protons (meson production), and magnetic fields (synchrotron radiation). The results are shown in figure 2, along with 


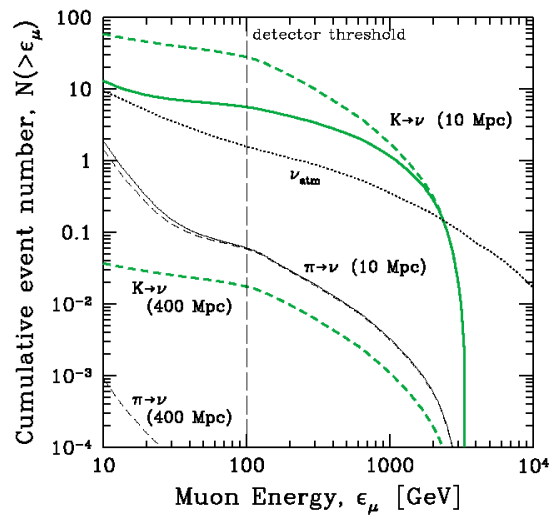

FIGURE 3. Cumulative muon event number from choked jets. Thin-black (thick-green) lines denote pion (kaon) contributions. Solid lines denote a mildly relativistic jet and a supernova distance of $10 \mathrm{Mpc}$. Dashed lines denote an ultrarelativistic jet and two supernova distances - $10 \mathrm{Mpc}$ and $400 \mathrm{Mpc}$ (labelled). Also shown is the atmospheric neutrino background over 1 day in a $3^{\circ}$ circle.

with acceleration time scale, at the illustrative radius $5 \times 10^{10} \mathrm{~cm}$. We find that protons may be accelerated to $\sim 70 \mathrm{TeV}$. This is sufficiently high for the production of neutrinos.

Protons near the highest energy ( $3-70 \mathrm{TeV})$ dominantly lose energy by photomeson production - we consider the pion and kaon mesons - while lower energy protons lose energy by pair production $(0.1-3 \mathrm{TeV})$ and collisions with target protons $(<0.1 \mathrm{TeV})$. Neutrinos are produced by the decays of mesons, so that the neutrino spectral shape is a modified version of the initial proton spectrum. The modification is dependent on the balance between the meson decay times and the meson energy-loss time scales. For example, at the highest energies, protons undergo photomeson production dominantly, so that the meson spectrum is similar to that of the proton. Then, mesons lose energy radiatively before they decay, so that the resulting neutrino spectrum is steeper than the initial meson spectrum by $\varepsilon^{-2}$. Similar arguments lead to the conclusion that the neutrino spectrum is steeper by $\varepsilon^{-1}$ at intermediate energies. We refer the reader to $[15,13]$ for detailed explanations and numerical values of spectral breaks.

\section{NEUTRINO DETECTABILITY}

Choked jets. In figure 3 we show the cumulative spectrum of neutrino induced muons, from a supernova with a choked jet. We adopt the fiducial choked jet described above. A neutrino detector with an effective area of $1 \mathrm{~km}^{2}$, i.e. an IceCube class detector utilizing upgoing muons, is considered. Thin and thick lines denote pion and kaon contributions, respectively. Three sets of supernovae are shown: a supernova at $10 \mathrm{Mpc}$ with a mildly relativistic jet (solid lines), a supernova at $10 \mathrm{Mpc}$ with a highly relativistic jet (dashed lines), and a supernova at $400 \mathrm{Mpc}$ with a highly relativistic jet (dashed lines labelled by "400 Mpc"). With a detector threshold of $100 \mathrm{GeV}$ (the vertical dashed line), 
the expected total event number for a mildly relativistic jet at $10 \mathrm{Mpc}$ is 6 . Adopting a steeper initila proton spectral index yields less events; $s=2.1(2.3,2.5)$ yields $4(2$, 1) events. In all jets, the majority of neutrinos come from kaons, because kaons are more massive than pions and suffer less cooling (the radiative cooling time scale is $\propto m^{4}$ ). Also, the kaon decay time is slightly shorter than the pion. Since the events arrive in $a \approx 34$ second time bin and $\sim 3^{\circ}$ angular bin, it allows very strong rejection of atmospheric neutrino backgrounds. We show in the figure the atmospheric neutrino background within 1 day and a $3^{\circ}$ bin for comparison. The expected neutrino signal from a choked mildly relativistic jet easily exceeds the atmospheric neutrino background.

We find that neutrino emission is favoured by relativistic, low luminosity and long duration jets. For example in figure 3 , a highly relativistic jet results in more events than a mildly relativistic jet (compared the $10 \mathrm{Mpc}$ cases; 28 events as opposed to 6 events). This is because the mesons experience less energy-loss at a given meson energy. Similar arguments can be made for the luminosity: a lower luminosity leads to a lower photon density and thus less meson cooling. In fact, in the reverse shock, meson cooling turns out to be more important than the linear scaling of events to the total energy. For example, a jet with total energy $10^{53} \mathrm{erg}\left(10^{54} \mathrm{erg}\right)$ yields 17 events ( 2 events) instead of 6 events, in contrary to a naive correlation with the total energy.

Mildly relativistic jets. For successful mildly relativistic jets, the neutrino detection prospects are better. Successful jets have neutrino emission from two sources, (i) the reverse shock, and (ii) internal shocks. We illustrate with the jet parameters $L_{\text {iso }}=$ $10^{52} \mathrm{erg} \mathrm{s}^{-1}, T_{j}=100 \mathrm{~s}$, and $\varepsilon_{e}=\varepsilon_{B}=0.1$. With these parameters, $t_{\mathrm{He}} \sim 43 \mathrm{~s}$, and the cold jet does indeed break out of the star.

For calculating the reverse shock emission we introduce an additional factor of $1 / 2$ to the neutrino fluence, because only about $\sim t_{\mathrm{He}} / T_{j} \sim 1 / 2$ of the jet length has been shocked by the reverse shock. Note that our chosen parameters have the equivalent $E_{\text {iso }}$ to a choked jet yielding 2 events. However, we expect $\sim(1 / 2)\left(2^{2} / 10^{-1}\right) \sim 20$ times more neutrino events, because $L_{\text {iso }}$ is a factor 10 smaller. Indeed, evaluating the muon event yields 36 events per mildly relativistic jet. However, internal shock emission is much larger. This is largely because the internal shock region has lower photon densities compared to the reverse shock region, and thus less particle cooling. Also, the internal shock is highly relativistic in the lab frame, so that particles are Lorentz boosted; the reverse shock in comparison is typically sub-relativistic. Using the above jet parameters, with a variability time scale of $0.01 \mathrm{~s}$, internal shocks typically occur at a radius of $\sim 2 \Gamma_{j}^{2} \delta t=6 \times 10^{10} \mathrm{~cm}$. We find neutrino events that are $\sim 100$ times larger than from the reverse shock.

Suernova rate. The key remaining issue is the fraction of core-collapse supernovae which have relativistic jets, and the core-collapse supernova rate. As we commented in the introduction, the presence of mildly relativistic jets has been suggested in supernovae, based on their late-time radio emission. The fraction of all core-collapse supernova with jets is perhaps $\sim 1 \%$, which is significantly larger than the fraction of GRBs. We note this is a lower limit, as it is conceivable that there is a distribution of jets, with lower Lorentz factor jets being more common. Choked jets are currently unconstrained. The rate of core-collapse supernovae in the local Universe is $\sim 1-3 \mathrm{yr}^{-1}$ [18]; 
examples of recent close supernovae can be found in Ref. [19]. The actual rate could be even higher, as a systematic survey of all nearby galaxies has not been performed to date. Considering that jet opening also follows a distribution, one could potentially test mildly relativistic jet formation in several decades. However, successful mildly relativistic jets would be beamed, so that detection prospects are suppressed by a geometrical amount. In either case, correlating with the optical signal of the supernova explosions would greatly help enhance the significance of the signal. Thus there are high prospects of detecting or constraining neutrinos from jet-like structures.

\section{DISCUSSION AND CONCLUSIONS}

We showed that core-collapse supernovae is a leading candidate of high-energy neutrino detection by $1 \mathrm{Km}^{3}$ scale neutrino detectors. We discussed hidden jets, which are dark in photons due to it being (i) mildly relativistic and hence optically thick to gamma rays, or (ii) failing to break out of the star. Both jets are bright in neutrinos and its neutrino emission can be detected from $<50 \mathrm{Mpc}$ and $<10 \mathrm{Mpc}$ away, respectively. The reason for the difference is that the choked jet emitting region is surrounded by a shocked medium, and results in stronger particle energy-loss, which suppresses the emission of high-energy neutrinos. The supernova rate in the local $10 \mathrm{Mpc}$ universe is at least $\sim 1-3$ $\mathrm{yr}^{-1}$, and neutrino detection could potentially be realized in the next few decades.

\section{REFERENCES}

1. T. Piran, Rev. Mod. Phys. 76, 1143 (2004) [arXiv:astro-ph/0405503].

2. P. Meszaros, Rept. Prog. Phys. 69, 2259 (2006) [arXiv:astro-ph/0605208].

3. S. E. Woosley, Astrophys. J. 405 (1993) 273.

4. A. MacFadyen and S. E. Woosley, Astrophys. J. 524, 262 (1999) [arXiv:astro-ph/9810274].

5. E. Berger, S. R. Kulkarni, D. A. Frail and A. M. Soderberg, Astrophys. J. 599, 408 (2003) [arXiv:astro$\mathrm{ph} / 0307228]$.

6. T. Totani, Astrophys. J. 598, 1151 (2003) [arXiv:astro-ph/0303621].

7. A. M. Soderberg, D. A. Frail and M. H. Wieringa, Astrophys. J. 607, L13 (2004) [arXiv:astro$\mathrm{ph} / 0402163]$.

8. M. H. P. van Putten, Astrophys. J. 611, L81 (2004) [ar]Xiv:astro-ph/0407112].

9. S. Campana et al., Nature 442, 1008 (2006) [arXiv:astro-ph/0603279].

10. B. E. Cobb, C. D. Bailyn, P. G. van Dokkum and P. Natarajan, Astrophys. J. 645, L113 (2006) [arXiv:astro-ph/0603832].

11. E. Pian et al., Nature 442, 1011 (2006) [arXiv:astro-ph/0603530].

12. A. M. Soderberg et al., Nature 442, 1014 (2006) [arXiv:astro-ph/0604389].

13. S. Horiuchi and S. Ando, Phys. Rev. D 77, 063007 (2008) [arXiv:0711.2580 [astro-ph]].

14. S. Razzaque, P. Meszaros and E. Waxman, Phys. Rev. Lett. 93, 181101 (2004) [Erratum-ibid. 94, 109903 (2005)] [arXiv:astro-ph/0407064].

15. S. Ando and J. F. Beacom, Phys. Rev. Lett. 95, 061103 (2005) [arXiv:astro-ph/0502521].

16. P. Meszaros and E. Waxman, Phys. Rev. Lett. 87, 171102 (2001) [arXiv:astro-ph/0103275].

17. A. Achterberg, Y. A. Gallant, J. G. Kirk and A. W. Guthmann, Mon. Not. Roy. Astron. Soc. 328, 393 (2001) [arXiv:astro-ph/0107530].

18. S. Ando, J. F. Beacom and H. Yuksel, Phys. Rev. Lett. 95, 171101 (2005) [arXiv:astro-ph/0503321].

19. M. D. Kistler, H. Yuksel, S. Ando, J. F. Beacom and Y. Suzuki, arXiv:0810.1959 [astro-ph]. 